frequency of appearance of any identical pentapeptide in both trichosanthin and ricin A to be only about 0.05 . In fact, three identical pentapeptides have been found. One is GYRAG in residues $80-84$ of trichosanthin and $83-87$ of ricin $\mathrm{A}$. The second is LRENI in residues $128-132$ of trichosanthin and $133-137$ of ricin $\mathrm{A}$. And the third is SEAAR in residues 166170 of trichosanthin and $176-180$ of ricin A. Thus the remarkable sequence homology between trichosanthin and ricin A seems not to be coincidental, and may well be of biological significance.

There is a pool of evidence indicating that it is an enzymatic process by which the toxic subunits of cytotoxins affect the host cell metabolism. For diphtheria toxin and cholera toxin, the subunit $\mathrm{A}$ acts on the elongation factor EF2 and GTP-binding protein respectively. Nevertheless they share a common mechanism $-\mathrm{ADP}=$ ribosylation. For plant toxins such as ricin and abrin, in spite of considerable efforts, the nature of their enzymatic activity is obscure. It is known, however, that they inactivate the $60 \mathrm{~S}$ subunit of ribosomes (for review see ref. 5). It is likely that different cytotoxins may not use the same mechanism, but the sequence homology between trichosanthin and ricin A shown here strongly suggests that these two do act by similar mechanisms. Taxonomically, Ricinus belongs to the Dicotyledoneae Archichlamydeae Euphorbiaceae Ricinus L. and Trichosanthes belongs to the Dicotyledoneae Sympetalae Cucurbitaceae Trichosanthea L. They are not closely related. But the possibility can not be ruled out that they originate from a common ancestor.

There is a speculation ${ }^{7}$ that in the ancient plants there was a special defensive protein which could inactivate eukaryotic ribosomes. In the evolution process, the gene coding for this toxic chain might have fused with the gene for some sort of sugarbinding proteins, resulting in cytotoxins with two different types of subunits as found in species like Ricinus. However, in some plants, such as pokeweed, the toxic chain remains independent and has been termed the ribosome-inactivating protein (RIP). The overall sequence homology between trichosanthin and ricin A strongly supports this hypothesis.

We thank Professor Wang Yu and his colleagues for their kindness in showing us the sequence of tricosanthin before publication. This work was supported by a special grant from the State Commission of Science and Technology of China.

Zhang XUEJUN WANG JiAHUAI

Biophysics Institute,

Academia Sinica,

Beijing, China

1. Acta zoologica sinica (Chinese) 22, 126-136 (1974).

. Gu Ziwei et al. Acta chemica sinica (Chinese) 43, 943-945 (1984).
. Wang Yu et al. (International Symposium on Organic Chemistry of Medicinal Natural Products) at Shanghai, China, November 1985

4. Pan Kezhen et al. Supplement of Proc. of China-Japan Bilateral symposium on Biophysics, May, 1985, Wuxi, China.

Olsnes, S. \& Pihl, A. in Molecular Action of Toxins and Viruses, 51-105 (Elsevier, Amsterdam, 1982).

6. Chan, W.Y., Tam, P.P. \& Yeung, H.W. Contraception 29 (1), 91-100 (1984).

Ready, M., Wilson, K., Piatak, M \& Robertus, J.D. J. biol. Chem. 259 (24), 15252-15256 (1984).

8. Lappi, D.A., Esch, F.S., Barbieri, L., Stirpe, F. \& Soria M Biochem. biophys. Res. Comm. 129, 934-942 (1985).

Kabsh, W. \& Sander, C. Proc. natn. Acad. Sci. U.S. A 81 1075-1078 (1984)

\section{Putting a charge on a quark}

SIR-The magnitudes of the quark electric charges raise the perplexing question: what is the magnitude of the unit electric charge? When the proton and electron were thought to be the elementary particles of nature, it appeared quite acceptable to take the electronic charge as the unit and to express all other charges in terms of it but, if quarks are elementary particles, it is meaningless to speak of a unit charge in any unambiguous way; only charge ratios are meaningful.

It is thus sensible to ask why the charge on the $u(u p)$ quark is minus twice that on the $d$ (down) quark and why the latter is one third that of the electron, but it is not meaningful to ask which of these is the unit charge. If it is desirable to work with integral charges, the $d$-charge should be taken as he unit; the $u$-charge is then -2 units and the electronic charge is +3 units. That the latter is universally accepted as the unit charge is merely a matter of a bias which perpetuates the belief that the electron is an elementary particle for which, at present there is no conclusive evidence.

Nothing in current particle theories even begins to answer the question why only two elementary charges exist in nature and why their ratio is -2 . Nor can these theories indicate how the answer is to be found. Quantum chromodynamics confuses things even more for it raises the additional question as to why all colours of a given quark flavour have the same electric charge.

If the three quarks in a nucleon form a linear structure, with one of the quarks always at the centre, as I have already proposed in my gravitational linear rotator model of the nucleon, then a simple variational principle answers the questions about quark charges.

In a series of papers I have proposed a linear rotator model of baryons in which the constituent particle triplets are gravitationally bound unitons, particles of mass $m \simeq(\hbar \mathrm{c} / G)^{1 / 2}$, where $G$ is the universal gravitational constant. The gravitational binding force between any two such particles is thus larger than the electrostatic force by a factor of the order of 137. Accepting this model one sees that the overall dynamical configuration is determined essentially by the gravitational field and that the electromagnetic field plays only a minor role in it; that is, the linear arrangement of three unitons, with one at the centre, is a consequence of the large masses of the unitons and would persist if the three unitons were electrically neutral. Nevertheless, the assignment of electric charges to the unitons does matter, for only one such assignment among the various possibilities is preferred by the energetics of the problem.

I now show that one obtains this unique uniton charge distribution by imposing the condition that the electrostatic potential energy of the linear triplet be a minimum. I have assumed here that gravity is the dominant force in this linear nucleon model, but any strong force field that gives the same linear arrangement will do.

If $Q$ is the charge on the nucleon and $q_{1}$, $q_{2}, q_{3}$, are the three quark charges, we have:

$$
q_{1}+q_{2}+q_{3}=Q
$$

where $q$ may be positive or negative. Placing $q_{1}$ at the centre of the linear configuration and $q_{2}$ and $q_{3}$ at the ends, the electrostatic potential energy is:

$$
E=\frac{1}{r}\left(q_{1} q_{2}+q_{1} q_{3}+\frac{1}{2} q_{2} q_{3}\right)
$$

where $2 r$ is the length of the structure; or,

$$
\begin{aligned}
r E & =q_{1}\left(q_{2}+q_{3}\right)+\frac{1}{2}\left(q_{2} q_{3}\right) \\
& =q_{1} Q-q_{1}^{2}+\frac{1}{2} Q q_{2}-\frac{1}{2} q_{1} q_{2}-\frac{1}{2} q_{2}^{2}
\end{aligned}
$$

where I have used (1) to eliminate $q_{3}$. Varying this with respect to $q_{2}$, keeping $Q$ and $q_{1}$ fixed, leads to the condition that $\mathrm{d}(r E) / \mathrm{d} q_{2}=0$ if, and only if, $q_{2}=q_{3}$.

In other words, regardless of the value of $Q$ and which uniton is at the centre of the configuration, the charges on the two ends must be equal in magnitude and in sign. Moreover, from (1), $q_{2}=1 / 2\left(Q-q_{1}\right)$ or $q_{2}=1 / 2\left(1-q_{1}\right)$ if $Q$ is the unit charge of the proton.

If, now, quark $q_{2}$ is at the mid point of this configuration, $q_{2}+2 q_{1}=Q^{\prime}$, where $Q^{\prime}$ is another value of the total charge, to be identified with that of the neutron, so that $q_{2}=-2 q_{1}$. But from the equivalent information for the proton $-2 q_{1}=1 / 2(1-$ $\left.q_{1}\right)$, or $\left(1+3 q_{1}\right)=0$ or $q_{1}=-1 / 3$ hence $q_{2}=$ $2 / 3 q_{1}$.

This is as far as one can go with this simple type of analysis, but its simplicity and its seeming meagreness hide its importance, for it clearly points to the linear rotatore with a uniton (quark) at its centre as the correct model of the nucleon. We are ineluctably led to this conclusion by the coefficient $-1 / 2$ in front of $q_{2}$ in equation (3); if it were numerically different, we would not obtain the important equality $q_{2}=q_{3}$.

LLOYd Motz

Department of Astronomy,

Columbia University,

New York 10027, USA 